\title{
Health-related quality of life, social support, and caregiver burden between six and 120 months after heart transplantation: a Spanish multicenter cross-sectional study
}

\author{
J.F. Delgado ${ }^{1}$, L. Almenar², F. González-Vilchez ${ }^{3}$, J.M. Arizón ${ }^{4}$, M. Gómez ${ }^{5}$, L.

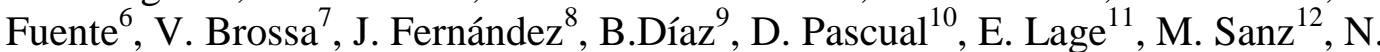 \\ Manito $^{13}$ and M.G. Crespo-Leiro ${ }^{14}$
}

${ }^{1}$ Hospital Universitario 12 de Octubre, Madrid, ${ }^{2}$ Hospital Universitario La Fe, Valencia, ${ }^{3}$ Hospital Universitario Marqués de Valdecilla, Santander, ${ }^{4}$ Hospital Universitario Reina Sofía, Córdoba, ${ }^{5}$ Hospital Universitario Puerta de Hierro, Madrid, ${ }^{6}$ Hospital Clínico Universitario de Valladolid, Valladolid, ${ }^{7}$ Hospital de la Santa Creu i Sant Pau, Barcelona, ${ }^{8}$ Hospital Universitario Gregorio Marañón, Madrid, ${ }^{9}$ Hospital Universitario Central de Asturias, Oviedo, ${ }^{10}$ Hospital Universitario Virgen de la Arrixaca, Murcia,

${ }^{11}$ Hospital Universitario Virgen del Rocío, Sevilla, ${ }^{12}$ Hospital Universitario Miguel Servet, Zaragoza, ${ }^{13}$ Hospital Universitario de Bellvitge, Barcelona, and ${ }^{14}$ Complejo Hospitalario de A Coruña, A Coruña, Spain

\begin{abstract}
A multicenter cross-sectional study was conducted to determine the current heart transplant (HTx) outcomes in Spain. Clinical and functional status, health-related quality of life (HRQoL), social support, and caregiver burden were analyzed in 303 adult transplant recipients (77.9\% males) living with one functioning graft. Mean age at time of HTx (SD) was 56.4 (11.4) years, and the reason for transplantation in all patients was congestive heart failure. All patients had received a first heart transplant $6( \pm 1), 12( \pm 2), 36( \pm 6), 60( \pm 10)$, or $120( \pm 20)$ months previously. Participants completed the Kansas City Cardiomyopathy Questionnaire (KCCQ), the EQ-5D, the Duke-UNC Functional Social Support Questionnaire, and the Zarit Caregiver Burden Scale. Reasonable HRQoL, social support, and caregiver burden levels were found at all time points, although a slight decrease in HRQoL was recorded at 120 months $(\mathrm{p} \leq 0.033)$. Multivariate regression analyses showed that complications, comorbidities, and hospitalizations were associated with HRQoL (EQ5D: $48.4 \%$ of explained variance, $F_{4,164}=38.46, \mathrm{p}<0.001$; KCCQ overall summary score: $45.0 \%$, $\left.F_{3,198}=54.073, \mathrm{p}<0.001\right)$. Patient functional capabilities and complications affected caregiver burden ( $\mathrm{p}<0.05$ ). In conclusion, HTx patients reported reasonable levels of HRQoL with low caregiver burden. Clinical variables related to these outcomes included functional status, complications, and number of admissions.
\end{abstract}

Key words: caregiver; comorbidity; heart transplantation; outcome assessment; quality of life 
The main goals of heart transplantation are to increase the life span of patients with advanced heart disease and to optimize their health-related quality of life (HRQoL), especially when these benefits cannot be guaranteed with the medical treatments available. The clinical effectiveness of heart transplantation is analyzed by comparing outcomes with those of other medical procedures, in terms of both survival rates (1-3) and patient-reported outcomes (PRO) (4). One of the most commonly used PRO measures is HRQoL, which can be defined as follows: "The value assigned to duration of life as modified by the impairments, functional states, perceptions, and social opportunities that are influenced by disease, injury, treatment, or policy (5)." Evidence from research has shown that patients' HRQoL after allograft improves in comparison with their situation before surgery (6-8). Furthermore, these benefits continue for as long as 5-10 yr posttransplant $(9,10)$. According to the HRQoL definition cited above, several factors can influence recipient HRQoL, including sociodemographic variables, work status, comorbidities, episodes of acute rejection, hospitalizations, side effects of medication, patient personality, and others (6), (1014). Thus, this study examined the effect of the following factors on patient HRQoL, six, 12, 36, 60, and 120 months after receiving the allograft: clinical and functional status of recipients, social support, work situation, employment rates, and caregiver burden. Furthermore, patient HRQoL was evaluated by applying disease-specific and generic patient-reported outcomes measures, as recommended in the guidelines (15), to adequately assess the impact of disease on HTx patients while allowing comparisons with other populations. This information is of interest, given the fact that, despite the excellent survival rates reported in Spain $(2,3)$, short and, especially, long-term results for variables related to patient HRQoL have not yet been comprehensively addressed.

\section{Methods}

\section{Study population}

Heart transplant patients attending programmed cardiology visits were invited to participate in this multicenter, epidemiological, cross-sectional study. Between December 2010 and December 2011, data were collected by cardiologists from the 14 reference centers for organ transplantation in Spain. Patients who agreed to participate and met the selection criteria were recruited: adult recipients (aged $\geq 18$ ) living with a single functioning graft, who had received a first heart transplant due to congestive heart failure, six $( \pm 1), 12( \pm 2), 36( \pm 6), 60( \pm 10)$, or $120( \pm 20)$ months before the interview. Multiple solid organ transplant or retransplant recipients were excluded. All patients were enrolled after providing written informed consent prior to the start of the study, which was conducted in accordance with the Declaration of Helsinki and Good Clinical Practice guidelines. The study protocol and case report form were approved by the Clinical Research Ethics Committee of the Hospital Universitario 12 de Octubre (Madrid, Spain). Data collection was conducted by a specially trained clinical monitor, under the supervision of the study coordinator (JFD). All data were entered in an electronic database installed in a secure server using numerical codes for the investigators and patients at each site. 


\section{Procedure}

Clinical data related to patient health status immediately before transplantation were retrieved from the medical records: age, sex, body mass index (BMI), heart disease that led to transplant (acute/chronic heart failure), indication (elective/urgent), and underlying heart disease (idiopathic dilated cardiomyopathy/ischemic cardiomyopathy/valvular cardiomyopathy/other).

Additionally, investigators evaluated the clinical status of participants at the time of the consultation, according to the following variables: New York Heart Association (NYHA) functional classification, left ventricular ejection fraction (by echocardiogram), resting heart rate, number of acute rejections recorded, number of biopsies after transplant, chronic oral anticoagulant treatment, number of hospital admissions (not scheduled), and infectious diseases (requiring medical treatment). The presence of coexisting complications or additional diseases was assessed by means of the Charlson Comorbidity Index. In addition, specific comorbidities were classified into several dichotomous variables ("yes" or "no"): high blood pressure; hyperlipidemia; malignancy (cutaneous malignancy, lymphomas, or other solid or hematological malignancies); and dermatological, urological, neuromuscular, and orthopedic diseases.

During the single study visit, patients were asked for information about their personal situation (living situation, marital status, work status, and educational level) at two time points: immediately before transplant and at the time of the interview. Satisfaction with their current work status was reported only by patients who were not retired at the time of the interview.

\section{Patient-reported outcomes}

Patient-reported outcomes were determined by the use of the following questionnaires:

\section{$E Q-5 D-3 L(16)$}

This generic instrument allows investigators to obtain valid information about patient HRQoL and community preference-based values (or utilities) related to all possible health states described $(n=243)$. The EQ-5D utility index in Spain ranges from -0.654 (worst possible health status) to 1 (completely healthy). Furthermore, the EQ-5D descriptive system encompasses five different domains (mobility, self-care, usual activities, pain/discomfort, and anxiety/depression). A visual analog scale (VAS) that ranges from 0 (worst state) to 100 (perfect health) is also used to determine patients' perceived HRQoL at the time of the interview.

\section{Kansas City Cardiomyopathy Questionnaire (KCCQ) (17)}

This instrument was developed to assess HRQoL in patients with chronic heart failure and has been successfully used in heart transplant patients. This disease-specific instrument comprises 23 items (five- to seven-point Likert scale items) assessing six domains: physical limitations, symptoms, symptom stability, social limitation, self-efficacy, and quality of life (QoL). In addition, two summary scores can be calculated: the overall summary score (OSS) and the clinical summary score (CSS). Domain scores can be easily converted into scales ranging from 0 (worst level) to 100 (highest level). 


\section{Duke-UNC Functional Social Support Questionnaire (18)}

This brief scale (11 items with five response levels) was developed with the purpose of measuring the strength of the respondent's social support network. Scores range from 11 (lowest level of social support) to 55 (highest level). A score of $\geq 32$ indicates a normal social network.

In addition, caregiver burden was assessed using Zarit Caregiver Burden Interview (19), a selfadministered instrument comprising 22 items on a five-point Likert scale. It was completed by the closest relative or caregiver of each patient, to determine the impact of the patient's condition on their own life and their feelings of stress or burden. Scoring ranges from 0 (lowest burden) to 88 (highest burden).

\section{Statistical analysis}

Participants were classified into five independent groups according to time since transplant: six $( \pm 1), 12( \pm 2), 36( \pm 6), 60( \pm 10)$, or $120( \pm 20)$ months, and a descriptive analysis of their sociodemographic and clinical variables was performed (frequencies and percentages for qualitative variables and measures of central tendency, dispersion and normality for quantitative variables). Differences in categorical variables among the groups were tested by applying the chisquare test or the Fisher's exact test in case of $2 \times 2$ tables or $<20$ valid cases. For quantitative variables, differences were compared using the Kruskal-Wallis test with the Mann-Whitney $U$ test (with Bonferroni's correction for multiple comparisons). Multivariate regression analyses (stepwise methods with enter and exit criteria of 0.05 and 0.1 , respectively) were carried out to study possible relationships between predictor (independent) variables (comorbidities and other clinical and sociodemographic variables) and criterion (dependent) variables (questionnaires: patient HRQoL, social support, and caregivers' feeling of burden). The following variables were entered in the models: time since transplant $(6 \pm 1,12 \pm 2,36 \pm 6,60 \pm 10$, or $120 \pm 20$ months), age, sex, marital status, living and work situation, educational level, presence of complications, NYHA classification, resting heart rate, number of acute rejections, number of biopsies after transplant, chronic oral anticoagulant treatment, number of unscheduled hospital admissions, and infectious diseases (requiring medical treatment). The possible multicollinearity between regression model parameters was considered by examining tolerance and the variance inflation factor.

The sample size was calculated with the aim of finding moderate differences in the outcome measures described above (effect size of 0.5) using pairwise comparisons among independent groups, with a Type I error of 0.05 and a power (1-ß) of 0.8 (one-tailed). Thus, the minimum sample size in each group was set at 51 patients. For two-tailed tests, a minimum of 64 patients per group would be required to have the same Type I error probability and statistical power.

SPSS (version 16.0) and Stata 10 (StataCorp, College Station, TX, USA) statistical packages were used to conduct all analyses. 


\section{Results}

\section{Sociodemographic and clinical characteristics}

A total of 350 heart transplant patients were formally invited to participate in the study, and data were collected from those who agreed to be interviewed and met the selection criteria $(\mathrm{n}=331,94.57 \%)$. However, after a quality control check of the database, a small proportion $(8.46 \%, \mathrm{n}=28)$ had to be withdrawn from the analysis because they do not meet the inclusion criteria in terms of time since transplant. Thus, a final sample of 303 transplant recipients (77.9\% males) with a mean age (SD) of 56.4 (11.4) yr was included in the analyses. As stated above, all participants were allocated to five different groups according to time since transplant. Moreover, 240 caregivers completed the Zarit Caregiver Burden Interview (97.3\% were close relatives and $2.7 \%$ professional caregivers). Sociodemographic and clinical variables are presented in Tables 13.

Table 1. Sample description: sociodemographic variables at interview time

\begin{tabular}{|c|c|c|c|c|c|c|c|c|c|c|c|}
\hline & \multicolumn{2}{|c|}{$\begin{array}{c}6 \text { months } \\
(\mathrm{n}=52)\end{array}$} & \multicolumn{2}{|c|}{$\begin{array}{l}12 \text { months } \\
(\mathrm{n}=57)\end{array}$} & \multicolumn{2}{|c|}{$\begin{array}{c}36 \text { months } \\
(\mathrm{n}=66)\end{array}$} & \multicolumn{2}{|c|}{$\begin{array}{l}60 \text { months } \\
(n=62)\end{array}$} & \multicolumn{2}{|c|}{$\begin{array}{l}120 \text { months } \\
(\mathrm{n}=66)\end{array}$} & \multirow{2}{*}{$\begin{array}{c}\mathrm{p} \\
\text { value }^{\mathrm{a}}\end{array}$} \\
\hline & $\mathrm{n}$ & $\%$ & $\mathrm{n}$ & $\%$ & $\mathrm{n}$ & $\%$ & $\mathrm{n}$ & $\%$ & $\mathrm{n}$ & $\%$ & \\
\hline \multicolumn{12}{|l|}{ Living situation } \\
\hline Alone & 3 & 5.8 & 4 & 7.0 & 12 & 18.2 & 3 & 4.8 & 7 & 10.6 & 0.021 \\
\hline Family & 47 & 90.4 & 53 & 93.0 & 54 & 81.8 & 58 & 93.5 & 59 & 89.4 & \\
\hline Institution & 2 & 3.8 & 0 & 0.0 & 0 & 0.0 & 0 & 0.0 & 0 & 0.0 & \\
\hline \multicolumn{12}{|l|}{ Marital status } \\
\hline Single & 8 & 15.4 & 8 & 14.0 & 5 & 7.6 & 7 & 11.3 & 3 & 4.5 & 0.213 \\
\hline Couple/Married & 39 & 75.0 & 42 & 73.7 & 48 & 72.8 & 52 & 83.9 & 55 & 83.3 & \\
\hline Widow/er & 2 & 3.8 & 2 & 3.5 & 2 & 3.0 & 0 & 0.0 & 4 & 6.1 & \\
\hline Separated/Divorced & 2 & 3.8 & 4 & 7.1 & 10 & 15.2 & 3 & 4.8 & 4 & 6.0 & \\
\hline \multicolumn{12}{|l|}{ Work status } \\
\hline Full time & 4 & 7.7 & 1 & 1.8 & 4 & 6.1 & 7 & 11.3 & 7 & 10.6 & 0.630 \\
\hline Part time & 3 & 5.8 & 3 & 5.3 & 1 & 1.5 & 2 & 3.2 & 0 & 0.0 & \\
\hline Retired & 4 & 7.7 & 9 & 15.8 & 8 & 12.1 & 9 & 14.5 & 5 & 7.6 & \\
\hline $\begin{array}{l}\text { Retired due to } \\
\text { disease }\end{array}$ & 31 & 59.6 & 38 & 66.7 & 42 & 63.6 & 36 & 58.1 & 41 & 62.1 & \\
\hline Unemployed & 8 & 15.4 & 6 & 10.5 & 10 & 15.2 & 7 & 11.3 & 8 & 12.1 & \\
\hline \multicolumn{12}{|l|}{ Employed } \\
\hline Yes & 7 & 13.5 & 4 & 7.0 & 5 & 7.6 & 9 & 14.5 & 7 & 10.6 & 0.571 \\
\hline
\end{tabular}

HTx, heart transplant.

${ }^{a}$ Chi-square test (exact test in case of $2 \times 2$ tables or in case of valid cases $<20$ ). 
Table 2. Clinical characteristics of heart recipients: categorical variables

\begin{tabular}{|c|c|c|c|c|c|c|c|c|c|c|c|}
\hline & \multicolumn{2}{|c|}{$\begin{array}{l}6 \text { months } \\
(\mathrm{n}=52)\end{array}$} & \multicolumn{2}{|c|}{$\begin{array}{l}12 \text { months } \\
(\mathrm{n}=57)\end{array}$} & \multicolumn{2}{|c|}{$\begin{array}{l}36 \text { months } \\
(\mathrm{n}=66)\end{array}$} & \multicolumn{2}{|c|}{$\begin{array}{l}60 \text { months } \\
(n=62)\end{array}$} & \multicolumn{2}{|c|}{$\begin{array}{l}120 \text { months } \\
(\mathrm{n}=66)\end{array}$} & \multirow{2}{*}{$\begin{array}{c}\mathrm{p} \\
\text { value }^{\mathrm{a}}\end{array}$} \\
\hline & $\mathrm{n}$ & $\%$ & $\mathrm{n}$ & $\%$ & $\mathrm{n}$ & $\%$ & $\mathrm{n}$ & $\%$ & $\mathrm{n}$ & $\%$ & \\
\hline \multicolumn{12}{|l|}{ Sex } \\
\hline Male & 37 & 71.2 & 48 & 84.2 & 51 & 77.3 & 49 & 79.0 & 51 & 77.3 & 0.597 \\
\hline \multicolumn{12}{|c|}{ Disease inducing HTx } \\
\hline Chronic & 46 & 88.5 & 54 & 94.7 & 63 & 95.5 & 55 & 88.7 & 61 & 92.4 & 0.534 \\
\hline Acute & 5 & 9.6 & 3 & 5.3 & 3 & 4.5 & 7 & 11.3 & 4 & 6.1 & \\
\hline Acute stroke & 2 & 3.8 & 2 & 3.5 & 3 & 4.5 & 4 & 6.5 & 6 & 9.1 & \\
\hline Inflam. disease & 3 & 5.8 & 0 & 0.0 & 3 & 4.5 & 2 & 3.2 & 1 & 1.5 & \\
\hline \multicolumn{12}{|l|}{ Type of HTx } \\
\hline Elective & 39 & 75.0 & 46 & 80.7 & 53 & 80.3 & 46 & 74.2 & 57 & 86.4 & 0.499 \\
\hline Urgent & 12 & 23.1 & 11 & 19.3 & 13 & 19.7 & 16 & 25.8 & 9 & 13.6 & \\
\hline \multicolumn{12}{|c|}{ Underlying heart disease at HTx } \\
\hline $\begin{array}{l}\text { Dilated } \\
\text { Idiopathic }\end{array}$ & 23 & 44.2 & 19 & 33.3 & 32 & 48.5 & 26 & 41.9 & 29 & 43.9 & 0.108 \\
\hline Ischemic & 18 & 34.6 & 24 & 42.1 & 17 & 25.8 & 20 & 32.3 & 32 & 48.5 & \\
\hline Valvular & 3 & 5.8 & 7 & 12.3 & 8 & 12.1 & 6 & 9.7 & 2 & 3.0 & \\
\hline Other & 8 & 15.4 & 7 & 12.3 & 7 & 10.6 & 10 & 16.1 & 2 & 3.0 & \\
\hline \multicolumn{12}{|c|}{ NYHA functional at interview } \\
\hline Class I & 45 & 86.5 & 48 & 84.2 & 58 & 87.9 & 53 & 85.5 & 42 & 63.6 & 0.005 \\
\hline Class $>\mathrm{I}$ & 7 & 13.5 & 9 & 15.8 & 8 & 12.1 & 9 & 14.5 & 24 & 36.4 & \\
\hline Class II & 7 & 13.5 & 7 & 12.3 & 5 & 7.6 & 8 & 12.9 & 22 & 33.3 & \\
\hline Class III & 0 & 0.0 & 2 & 3.5 & 3 & 4.5 & 1 & 1.6 & 2 & 3.0 & \\
\hline \multicolumn{12}{|c|}{ NYHA objective at interview } \\
\hline Class A & 42 & 80.8 & 47 & 82.5 & 55 & 83.3 & 52 & 83.9 & 44 & 66.7 & 0.065 \\
\hline Class $>\mathrm{A}$ & 10 & 19.2 & 10 & 17.5 & 11 & 16.7 & 9 & 14.5 & 22 & 33.3 & \\
\hline \multicolumn{12}{|c|}{ Resting heart rate at interview } \\
\hline S. Tachycardia & 41 & 78.8 & 48 & 84.2 & 48 & 72.7 & 55 & 88.7 & 52 & 78.8 & 0.348 \\
\hline Rhythm P. & 1 & 1.9 & 1 & 1.8 & 4 & 6.1 & 1 & 1.6 & 5 & 7.6 & \\
\hline \multicolumn{12}{|c|}{ Immunosuppressive treatment: Steroids have been withdrawn (at interview) } \\
\hline Yes & 1 & 1.9 & 8 & 14.0 & 15 & 22.7 & 12 & 19.4 & 21 & 31.8 & 0.001 \\
\hline \multicolumn{12}{|c|}{ Severe GVD at interview } \\
\hline Yes & 0 & 0.0 & 1 & 1.8 & 4 & 6.1 & 2 & 3.2 & 7 & 10.6 & 0.042 \\
\hline \multicolumn{12}{|c|}{ Pacemaker at interview } \\
\hline Yes & 1 & 1.9 & 3 & 5.3 & 4 & 6.1 & 7 & 11.3 & 11 & 16.7 & 0.036 \\
\hline \multicolumn{12}{|c|}{ Anticoagulant $\left(\right.$ Sintrom $\left.^{\circledR}\right)$ at interview } \\
\hline Yes & 4 & 7.7 & 1 & 1.8 & 4 & 6.1 & 7 & 11.3 & 4 & 6.1 & 0.340 \\
\hline \multicolumn{12}{|c|}{ Comorbidities at interview } \\
\hline \multicolumn{12}{|c|}{ AHT } \\
\hline Yes & 22 & 42.3 & 30 & 52.6 & 42 & 63.6 & 42 & 67.7 & 48 & 72.7 & 0.005 \\
\hline \multicolumn{12}{|l|}{ Hyperlipidemia } \\
\hline Yes & 26 & 50.0 & 30 & 52.6 & 34 & 51.5 & 35 & 56.5 & 40 & 60.6 & 0.769 \\
\hline Dermatological & sease & & & & & & & & & & \\
\hline Yes & 1 & 1.9 & 3 & 5.3 & 3 & 4.5 & 3 & 4.8 & 6 & 9.1 & 0.513 \\
\hline Diabetes mellitu & & & & & & & & & & & \\
\hline Yes & 16 & 30.8 & 17 & 29.8 & 17 & 25.8 & 13 & 21.0 & 29 & 43.9 & 0.061 \\
\hline Urological disea & & & & & & & & & & & \\
\hline Yes & 1 & 1.9 & 4 & 7.0 & & & 3 & 4.8 & 6 & 9.1 & 0.098 \\
\hline
\end{tabular}


Table 2. Clinical characteristics of heart recipients: categorical variables

\begin{tabular}{|c|c|c|c|c|c|c|c|c|c|c|c|}
\hline & \multicolumn{2}{|c|}{$\begin{array}{l}6 \text { months } \\
(n=52)\end{array}$} & \multicolumn{2}{|c|}{$\begin{array}{l}12 \text { months } \\
(\mathrm{n}=57)\end{array}$} & \multicolumn{2}{|c|}{$\begin{array}{c}36 \text { months } \\
(\mathrm{n}=66)\end{array}$} & \multicolumn{2}{|c|}{$\begin{array}{l}60 \text { months } \\
(\mathrm{n}=62)\end{array}$} & \multicolumn{2}{|c|}{$\begin{array}{l}120 \text { months } \\
\quad(\mathrm{n}=66)\end{array}$} & \multirow{2}{*}{$\begin{array}{c}\mathrm{p} \\
\text { value }^{\mathrm{a}}\end{array}$} \\
\hline & $\mathrm{n}$ & $\%$ & $\mathrm{n}$ & $\%$ & $\mathrm{n}$ & $\%$ & $\mathrm{n}$ & $\%$ & $\mathrm{n}$ & $\%$ & \\
\hline \multicolumn{12}{|c|}{ Neuromuscular disease } \\
\hline Yes & 7 & 13.5 & 5 & 8.8 & 4 & 6.1 & 5 & 8.1 & 8 & 12.1 & 0.661 \\
\hline \multicolumn{12}{|c|}{ Neoplasm (cutaneous neoplasm, lymphomas, or other solid or hematological malignancies) } \\
\hline Yes & 0 & 0.0 & 1 & 1.8 & 4 & 6.1 & 4 & 6.5 & 11 & 16.7 & 0.002 \\
\hline \multicolumn{12}{|c|}{ Orthopedic diseases } \\
\hline Yes & 2 & 3.8 & 3 & 5.3 & 6 & 9.1 & 7 & 11.3 & 12 & 18.2 & 0.068 \\
\hline
\end{tabular}

HTx, heart transplant; SD, standard deviation.

GVD, graft vascular disease: patient has required a surgical or percutaneous revascularization, or has generated a ventricular dysfunction, or has produced heart failure or angina.

${ }^{a}$ Chi-square test (exact test in case of $2 \times 2$ tables or in case of valid cases $<20$ ).

Table 3. Clinical characteristics of heart recipients: continuous variables

\begin{tabular}{|c|c|c|c|c|c|c|c|c|c|c|c|}
\hline & \multicolumn{2}{|c|}{$\begin{array}{c}6 \text { months } \\
(\mathrm{n}=52)\end{array}$} & \multicolumn{2}{|c|}{$\begin{array}{c}12 \text { months } \\
(\mathrm{n}=57)\end{array}$} & \multicolumn{2}{|c|}{$\begin{array}{l}36 \text { months } \\
(\mathrm{n}=66)\end{array}$} & \multicolumn{2}{|c|}{$\begin{array}{l}60 \text { months } \\
(\mathrm{n}=62)\end{array}$} & \multicolumn{2}{|c|}{$\begin{array}{l}120 \text { months } \\
(\mathrm{n}=66)\end{array}$} & \multirow{2}{*}{$\begin{array}{c}\mathrm{p} \\
\text { value }^{\mathrm{a}}\end{array}$} \\
\hline & Mean & SEM & Mean & SEM & Mean & SEM & Mean & SEM & Mean & SEM & \\
\hline Age at HTx & $53.49^{\mathrm{b}}$ & 1.56 & 57.04 & 1.18 & 55.69 & 1.35 & 55.02 & 1.69 & 60.20 & 1.38 & 0.006 \\
\hline BMI at HTx & 25.27 & 0.50 & 25.40 & 0.54 & 25.87 & 0.49 & 25.23 & 0.48 & 25.81 & 0.51 & 0.872 \\
\hline $\begin{array}{l}\text { Left ventricular ejection } \\
\text { fraction at HTx (Eco) }(\%)\end{array}$ & 64.92 & 1.02 & 66.34 & 1.14 & 64.67 & 1.22 & 66.28 & 1.05 & 64.00 & 1.04 & 0.601 \\
\hline $\begin{array}{l}\text { No. hospital admissions after } \\
\text { HTx (>24 hrs not scheduled) }\end{array}$ & $0.32^{\mathrm{b}}$ & 0.08 & $0.83^{b}$ & 0.14 & $1.09^{b}$ & 0.20 & $1.03^{b}$ & 0.23 & 2.09 & 0.29 & 0.001 \\
\hline $\begin{array}{l}\text { No. infections requiring } \\
\text { hospitalization after HTx }\end{array}$ & 0.23 & 0.08 & 0.54 & 0.12 & 0.45 & 0.09 & 0.44 & 0.13 & 0.65 & 0.14 & 0.049 \\
\hline $\begin{array}{l}\text { No. episodes of rejection after } \\
\text { HTx }\end{array}$ & $0.21^{\mathrm{b}}$ & 0.06 & $0.61^{b}$ & 0.16 & $0.48^{b}$ & 0.10 & 0.80 & 0.15 & 1.42 & 0.19 & 0.001 \\
\hline $\begin{array}{l}\text { Charlson Index at time of } \\
\text { interview (mean, SD) }\end{array}$ & $0.50^{\mathrm{b}}$ & 0.75 & $0.73^{b}$ & 1.11 & $0.71^{b}$ & 1.47 & $0.64^{b}$ & 1.02 & 1.42 & 1.40 & 0.010 \\
\hline
\end{tabular}

SEM, standard error of the mean; HTx, heart transplant.

${ }^{a}$ Kruskal-Wallis test.

${ }^{b}$ Bonferroni's correction for $2 \times 2$ comparisons (significant differences with 120 months group).

Overall, there were no differences in sociodemographic or economic variables. Regarding their clinical profiles, patients had chronic heart disease before HTx (>88\% in all groups) and received an elective HTx (>74\% in all groups). The majority of patients had no limitations in physical activity, and there was no objective evidence of cardiovascular disease (NYHA Class I and A, respectively). Nevertheless, according to this scale, the percentage of patients with functional limitations increased significantly 120 months post-transplant (Table 2). With respect to the most relevant comorbidities, more complications were observed (particularly arterial hypertension and diabetes) in the group of patients who had received the allograft 120 months previously (Table 2). 
In addition, mean Charlson Index scores were significantly higher in this group, while patients remained fairly stable between 12 and 60 months post-transplant (Table 2).

\section{Reported outcomes and multivariate analysis}

The results showed reasonably good levels of patient functioning, well-being, and social support (Table 4) at all stages after transplant. No statistically significant differences were found between the different regions in Spain for EQ-5D utility scores $\left(F_{3,300}=1.219, \mathrm{p}=0.303\right)$.

Table 4. Measures of HRQoL, social support, and satisfaction with work status [at interview time]

\begin{tabular}{|c|c|c|c|c|c|c|c|c|c|c|c|}
\hline & \multicolumn{2}{|c|}{6 months } & \multicolumn{2}{|c|}{12 months } & \multicolumn{2}{|c|}{36 months } & \multicolumn{2}{|c|}{60 months } & \multicolumn{2}{|c|}{120 months } & \multirow{2}{*}{$\begin{array}{c}\mathrm{p} \\
\text { value }\end{array}$} \\
\hline & Mean & SEM & Mean & SEM & Mean & SEM & Mean & SEM & Mean & SEM & \\
\hline \multicolumn{12}{|l|}{ KCCQ [HRQoL] (0: worst-100: best) } \\
\hline Symptom frequency & 87.82 & 2.25 & 89.00 & 2.21 & 90.34 & 2.10 & 87.74 & 2.50 & 83.43 & 2.69 & 0.104 \\
\hline Symptom stability & 60.10 & 2.68 & 54.39 & 2.18 & 54.92 & 2.24 & 54.17 & 2.16 & 59.62 & 2.61 & 0.237 \\
\hline Impact of symptoms & 88.94 & 2.26 & 90.92 & 1.88 & $93.43^{b}$ & 1.73 & 88.17 & 2.26 & $82.44^{\mathrm{b}}$ & 2.77 & 0.010 \\
\hline Global symptoms & 78.95 & 1.63 & 78.01 & 1.47 & 79.57 & 1.45 & 76.83 & 1.86 & 75.16 & 2.07 & 0.511 \\
\hline Quality of life & $77.72^{b}$ & 2.99 & 79.82 & 2.73 & $88.57^{\mathrm{b}}$ & 1.59 & 85.35 & 2.39 & 80.51 & 2.40 & 0.017 \\
\hline Social limitation & 81.09 & 3.23 & 84.65 & 2.76 & $91.57^{\mathrm{b}}$ & 2.05 & 81.18 & 3.09 & $78.74^{\mathrm{b}}$ & 2.88 & 0.007 \\
\hline Physical limitation & 83.83 & 2.68 & 84.12 & 2.80 & 88.57 & 2.51 & 83.05 & 3.23 & 78.35 & 2.80 & 0.005 \\
\hline Self-efficacy & 87.50 & 2.85 & 85.31 & 2.22 & 89.39 & 1.97 & 84.68 & 2.10 & 84.42 & 2.59 & 0.207 \\
\hline OSS & 80.46 & 2.16 & 81.65 & 1.92 & $87.05^{\mathrm{b}}$ & 1.30 & 81.60 & 2.14 & $78.27^{\mathrm{b}}$ & 1.94 & 0.030 \\
\hline CSS & 81.39 & 1.80 & 81.06 & 1.77 & $84.11^{\mathrm{b}}$ & 1.54 & 79.94 & 2.19 & $76.79^{\mathrm{b}}$ & 1.89 & 0.026 \\
\hline EQ-5D utility index & 0.81 & 0.03 & 0.82 & 0.03 & 0.85 & 0.03 & $0.86^{\mathrm{b}}$ & 0.02 & $0.75^{\mathrm{b}}$ & 0.03 & 0.033 \\
\hline EQ-5D VAS (0-100) & $79.04^{\mathrm{b}}$ & 2.01 & 76.35 & 2.18 & $79.48^{\mathrm{b}}$ & 1.68 & 75.34 & 2.43 & $68.31^{\mathrm{b}}$ & 2.46 & 0.011 \\
\hline Duke-UNC [Social Support] (11-55) & 46.77 & 0.97 & 46.55 & 1.12 & 46.36 & 0.84 & 46.36 & 0.89 & 45.13 & 1.03 & 0.765 \\
\hline $\begin{array}{l}\text { Satisfaction with working status (VAS, } 0- \\
10)^{\mathrm{c}}\end{array}$ & 7.07 & 0.79 & 5.47 & 0.82 & 6.31 & 1.18 & 5.71 & 0.76 & 6.29 & 0.81 & 0.638 \\
\hline
\end{tabular}

SEM, standard error of the mean; OSS, overall status summary; CSS, clinical summary score.

${ }^{a}$ Kruskal-Wallis test.

${ }^{b}$ Bonferroni's correction for $2 * 2$ comparisons.

${ }^{c}$ Satisfaction with working status was assessed only by patient who were active (not retired due to disease or age) at interview time (total subsample, $\mathrm{n}=79$ ).

However, a slightly higher proportion of patients reported some difficulties in the EQ-5D domains at 120 months post-transplant (Fig. 1). Caregiver burden was low in all groups (mean scores and standard error of the mean, by group): six months: 16.24 (1.61); 12 months: 18.41 (1.58); 36 months: 16.57 (1.87); 60 months: 14.49 (1.95); and 120 months: 17.07 (1.59); $\mathrm{p}=0.278$, in the Kruskal-Wallis test. 


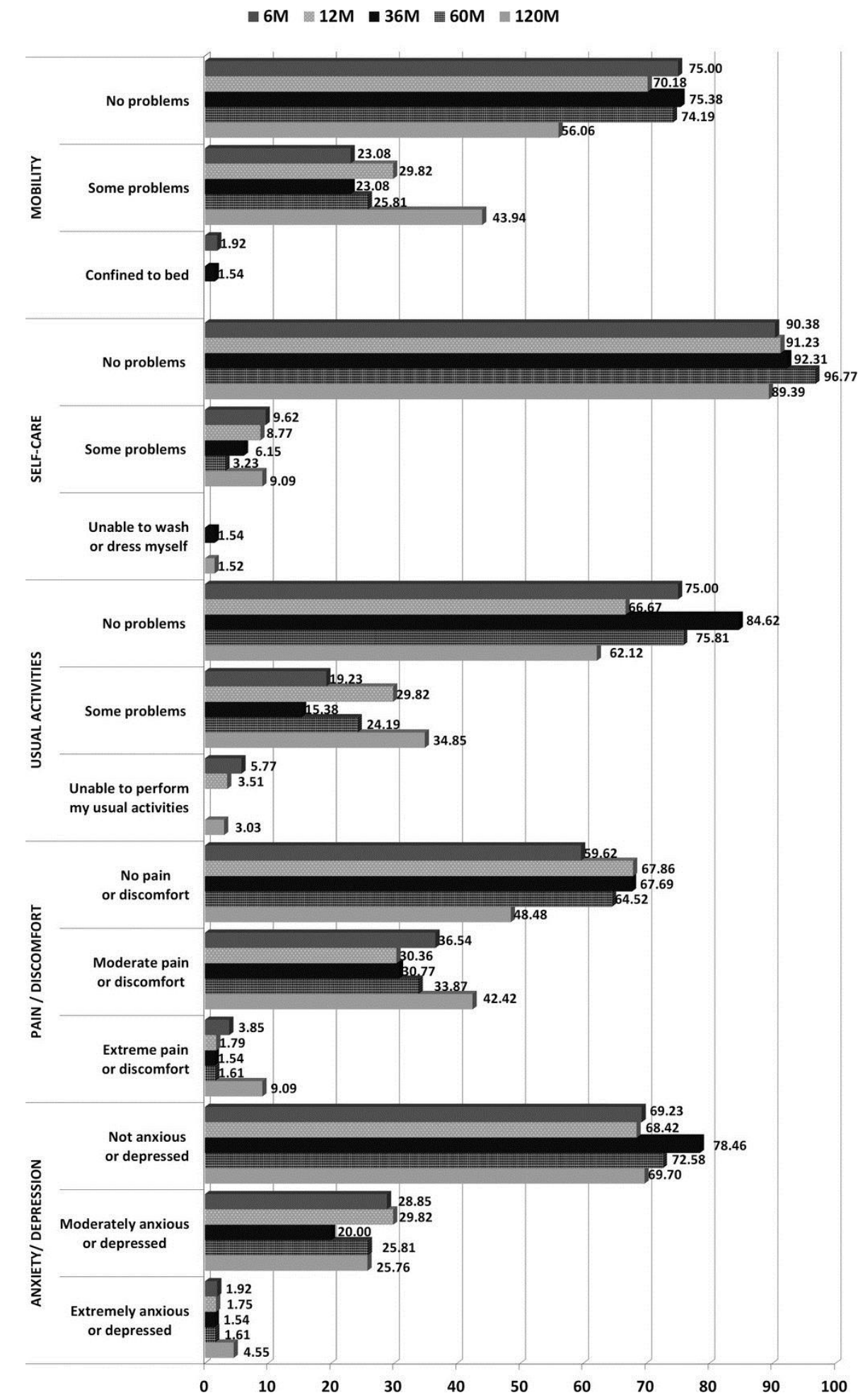

Figure 1 Domains of EQ-5D by study groups. The percentage of patients at all stages after transplant showed good punctuation at EQ-5D (five domains). However, a slightly higher proportion of patients suggested some difficulties in the EQ-5D domains at 120 months post-transplant. 
The relationship of clinical and sociodemographic variables with the scores of the instruments detailed in Table 4 was also assessed in multivariate analyses to control the possible effect of confounding factors (Table 5). With respect to the EQ-5D utility index and the general domains of the KCCQ scores (EQ-5D utility index: $48.4 \%$ of explained variance, $F_{4,164}=38.46, \mathrm{p}<0.001$ and OSS: $45 \%$ of explained variance, $F_{3,198}=54.073, \mathrm{p}<0.001$ ), the most related factors were comorbidities (neuromuscular and urological disease), hospital admissions, and severe graft vascular disease (GVD). All these parameters were negatively associated with utilities and HRQoL scores. Female sex was also negatively related to EQ-5D values, but to a lesser degree.

Table 5. Variables associated with patients' well-being and caregivers' burden

\begin{tabular}{|c|c|c|c|c|c|c|}
\hline & \multirow{2}{*}{$\mathrm{B}$} & \multicolumn{2}{|c|}{ CI of B } & \multirow{2}{*}{ Beta } & \multirow{2}{*}{$\mathrm{p}$ value } & \multirow{2}{*}{ Tolerance } \\
\hline & & Minimum & Maximum & & & \\
\hline \multicolumn{7}{|c|}{ EQ-5D utility index, $R^{2}=0.484, F_{4,164}=38.46, \mathrm{p}<0.001$} \\
\hline OSS & 0.008 & 0.007 & 0.010 & 0.585 & 0.000 & 0.976 \\
\hline Neuromuscular disease & -0.158 & -0.240 & -0.075 & -0.218 & 0.000 & 0.949 \\
\hline Urological disease & -0.183 & -0.301 & -0.066 & -0.174 & 0.002 & 0.988 \\
\hline Sex & -0.076 & -0.136 & -0.016 & -0.142 & 0.014 & 0.977 \\
\hline \multicolumn{7}{|c|}{ KCCQ-OSS, $R^{2}=0.450, F_{3,198}=54.073, \mathrm{p}<0.001$} \\
\hline EQ-5D utility index & 43.105 & 35.557 & 50.653 & 0.608 & 0.000 & 0.953 \\
\hline GVD & -10.198 & -18.219 & -2.178 & -0.143 & 0.013 & 0.853 \\
\hline Admissions to hospital & -1.177 & -2.243 & -0.112 & -0.127 & 0.031 & 0.818 \\
\hline \multicolumn{7}{|c|}{ Zarit Caregivers' Burden, $R^{2}=0.225, F_{5,168}=9.44, \mathrm{p}<0.001$} \\
\hline OSS & -0.178 & -0.280 & -0.076 & -0.248 & 0.001 & 0.922 \\
\hline Infections requiring hospitalization & 2.844 & 1.024 & 4.665 & 0.218 & 0.002 & 0.957 \\
\hline Biopsies post-transplant & -0.646 & -1.019 & -0.273 & -0.243 & 0.001 & 0.946 \\
\hline Resting heart rate & 2.883 & 0.580 & 5.186 & 0.172 & 0.014 & 0.984 \\
\hline Duke-UNC scale & -0.299 & -0.545 & -0.053 & -0.173 & 0.017 & 0.916 \\
\hline \multicolumn{7}{|c|}{ Duke-UNC [Social support], $R^{2}=0.144, F_{5,168}=5.49, \mathrm{p}<0.001$} \\
\hline OSS & 0.079 & 0.015 & 0.142 & 0.190 & 0.016 & 0.872 \\
\hline Biopsies post-transplant & -0.354 & -0.582 & -0.126 & -0.230 & 0.003 & 0.935 \\
\hline Zarit Caregivers' Burden & -0.112 & -0.201 & -0.023 & -0.193 & 0.014 & 0.856 \\
\hline Use of Sintrom & -3.894 & -7.367 & -0.420 & -0.164 & 0.028 & 0.955 \\
\hline NYHA objective & 1.488 & 0.009 & 2.968 & 0.149 & 0.049 & 0.928 \\
\hline
\end{tabular}

OSS, overall status summary; GVD, graft vascular disease; CI, confidence intervals.

In contrast, there was no association between time since transplantation and health status. Regarding patients' perception of their social network, a very small proportion of variance in the Duke scale could be explained by independent variables $(14.4 \%)$. Finally, considering caregiver burden $\left(22.5 \%\right.$ of explained variance, $\left.F_{5,168}=9.44, \mathrm{p}<0.001\right)$, the main clinical variables related to higher caregiver burden were the total number of infections requiring hospitalization and resting heart rate; while OSS scores, number of biopsies, and Duke scale scores were negatively related to burden scores. 


\section{Discussion}

In line with the recent recommendations for measuring HRQoL in cardiovascular diseases (20), a complete evaluation of patients at six, 12, 36, 60, and 120 months post-transplant was carried out by applying generic and disease-specific instruments.

Overall, the results showed reasonably high levels of patient HRQoL and functioning capabilities at all stages post-transplant. Compared with previous studies conducted in Spain, our findings at six and 12 months post-transplant for the KCCQ questionnaire (both CSS and OSS domains) were similar to those published by Ortega et al. (17) in heart recipients. Furthermore, mean EQ-5D VAS scores obtained from our series and that of Ortega et al. (17) were also compatible. Regarding later post-transplantation stages, the high levels of HRQoL reported by transplant recipients in our study are consistent with the results from other studies, which reported generally stable transplant benefits in terms of high HRQoL satisfaction in patients at 60, 72, and 120 months post-transplant $(9,21)$. However, we found that some difficulties were reported in the EQ-5D domains 120 months post-transplant. In fact, the VAS values recorded throughout this study were similar to those reported in the general Spanish population (22) in all groups, with the exception of the 120 months post-transplant assessment, where scores were lower $(23,24)$

After controlling for possible confounding factors in the multivariate analyses, this decrease in patient well-being was not related to age or time since transplant, but rather to the presence of comorbid medical conditions, severe GVD, and use of resources (number of hospitalizations). Once again, similar conclusions have been reached in previous studies from other developed countries $(9,10,25,26)$.

Patient social functioning was adequate, caregiver burden was low, and no differences between groups were found in these outcomes. Heart transplantation has been associated in the literature with a higher risk of anxiety and depressive disorders in caregivers 36 months after their family member's heart transplantation (27), probably exacerbated by uncertainty about the future, economic problems, and other causes (28). However, in our series, the burden felt by close relatives and professional caregivers was low in all groups. In this case, the significant parameters related to this burden included recipient HRQoL, the presence of complications, and social support.

This study has a number of limitations that should be addressed. First, it should be noted that all outcome measurements were obtained from transplant recipients and their caregivers who were willing to participate and complete the questionnaires. Thus, the results obtained from this sample may be biased in terms of overestimation or underestimation of HRQoL, social support, and caregiver burden. Moreover, the results from the caregiver sample should be treated with caution because professional and family caregivers were combined and the basic demographic data of this group were not collected. Nevertheless, our results in the early stages are similar to those of a longitudinal study also conducted in Spain which followed transplant recipients up to 12 months post-transplant (17). We also appreciate that a longitudinal design would be preferable in order to take a closer look at the effect of clinical and sociodemographic variables on dependent variables, to quantify the magnitude of effect size, and to produce a methodologically more robust test of the stability of perceived benefits. Finally, although the total number of participants was relatively large, the smaller size of the subgroups may limit generalization of the results. Nevertheless, results at early post-transplantation stages were consistent with previous publications in our country, and the reasonable levels of well-being reported at later post-transplantation stages were congruent with the evidence of stability from previous longitudinal studies $(9,21)$. Moreover, various variables that were identified as predictive factors of patient HRQoL in other studies (i.e., comorbid medical conditions, severe GVD, hospitalizations) (10) were also associated with dependent variables in our setting. 
In conclusion, outcomes in Spain at all stages after heart transplant seem to be satisfactory, and recipients report reasonable levels of HRQoL and social support. Accordingly, their caregivers or relatives also report low levels of disruption in their life.

\section{Acknowledgements}

This study received funding from Astellas Pharma, Spain, and from the Spanish RIC Cardiovascular Research Network (Instituto de Salud Carlos III, Ministry of Economy and Competiveness). However, the authors' independence in designing the study, interpreting the data, and drafting and publishing the report were guaranteed in funding agreement. Finally, the authors are grateful to Jesus Cuervo (LASER Analytica, Spain) for his technical support in the design of this research.

\section{Authors' contributions}

J.F. Delgado: Contributed to study design, data collection, interpretation of the results, drafting the paper, and critical review of the manuscript; J. Fernandez, B. Díaz, D. Pascual, E. Lage, M. Sanz, and N. Manito: Contributed to study design, data collection, interpretation of the results, and critical review of the manuscript; M.G. Crespo-Leiro, L. Almenar, F. González-Vilchez, J.M. Arizón, M. Gómez-Bueno, L. De la Fuente, and S. Mirabet: Contributed to data collection, interpretation of the results, and critical review of the manuscript.

\section{References}

1. Scientific Registry of Transplant Recipients. National Report: Heart Summary. www ustransplant org on 01/12/2010 2010 December 1 Available from: URL: http://www.ustransplant.org/default.aspx

2. Almenar, L, Segovia, J, Crespo-Leiro, MG et al. Spanish Heart Transplantation Registry. 21st Official Report of the Spanish Society of Cardiology Working Group on Heart Failure and Heart Transplantation (1984-2009). Rev Esp Cardiol 2010: 63: 1317.

3. Almenar, L, Segovia, J, Crespo-Leiro, MG et al. Spanish Registry on Heart Transplantation. 22nd official report of the Spanish Society of Cardiology Working Group on Heart Failure and Heart Transplantation (1984-2010). Rev Esp Cardiol 2011: 64: 1138.

4. Saeed, I, Rogers, C, Murday, A. Health-related quality of life after cardiac transplantation: results of a UK national survey with norm-based comparisons. J Heart Lung Transplant 2008: 27: 675.

5. Patrick, DL, Erickson, P. Assessing health-related quality of life for clinical decision making. In: RM Rosser, SR Walker eds. Quality of Life: Assessment and Application. Lancaster, UK: MTP Press, 1988: 22.

6. Jalowiec, A, Grady, KL, White-Williams, C. Functional status one year after heart transplant. $J$ Cardiopulm Rehabil Prev 2007: 27: 24.

7. Martin-Rodriguez, A, Perez-San-Gregorio, MA, Az-Dominguez, R, Perez-Bernal, J. Healthrelated quality of life evolution in patients after heart transplantation. Transplant Proc 2008: 40: 3037.

8. Almenar-Pertejo, M, Almenar, L, Martinez-Dolz, L et al. Study on health-related quality of life in patients with advanced heart failure before and after transplantation. Transplant Proc 2006: 38: 2524.

9. Grady, KL, Naftel, DC, Kobashigawa, J et al. Patterns and predictors of quality of life at 5 to 10 years after heart transplantation. J Heart Lung Transplant 2007: 26: 535.

10. Grady, KL, Naftel, DC, Young, JB et al. Patterns and predictors of physical functional disability at 5 to 10 years after heart transplantation. J Heart Lung Transplant 2007: 26: 1182.

11. Grady, KL, Naftel, DC, Kirklin, JK et al. Predictors of physical functional disability at 5 to 6 years after heart transplantation. J Heart Lung Transplant 2005: 24: 2279. 
12. White-Williams, C, Jalowiec, A, Grady, K. Who returns to work after heart transplantation? J Heart Lung Transplant 2005: 24: 2255.

13. Grady, KL, Jalowiec, A, White-Williams, C. Patient compliance at one year and two years after heart transplantation. J Heart Lung Transplant 1998: 17: 383.

14. White-Williams, C, Wang, E, Rybarczyk, B, Grady, KL. Factors associated with work status at 5 and 10 years after heart transplantation. Clin Transplant 2011: 25: 599.

15. European Network for Health Technology Assessment E. Guideline. Endpoints used for relative effectiveness assessment of pharmaceuticals: Health-related quality of life and utility measures. 2013. EUNETHTA

16. Badia, X, Roset, M, Montserrat, S, Herdman, M, Segura, A. La versión española del EuroQol: descripción y aplicaciones. Med Clin (Barc) 1999: 112: 79.

17. Ortega, T, az-Molina, B, Montoliu, MA et al. The utility of a specific measure for heart transplant patients: reliability and validity of the Kansas City Cardiomyopathy Questionnaire. Transplantation 2008: 86: 804

18. Bellon, JA, Delgado, A, Luna, J, Lardelli, P. Validez y fiabilidad del cuestionario de apoyo social funcional Duke-UNC-11. Aten Primaria 1996: 18: 153.

19. Martín, M, Salvadó, I, Nadal, S, Miji, LC, Rico, JM, Lanz y Taussing, MI. Adaptación para nuestro medio de la Escala de Sobrecarga del Cuidador (Caregiver Burden Interview) de Zarit. Revista de Gerontología 1996: 6: 338

20. Mackintosh, A, Gibbons, E, Fitzpatrick, R. A Structured Review of Patient-Reported Outcomes Measures (PROMs) for Heart Failure: An Update 2009. Patient-reported Outcome Measurement Group: University of Oxford, 2009.

21. Grady, KL, Naftel, DC, White-Williams, C et al. Predictors of quality of life at 5 to 6 years after heart transplantation. J Heart Lung Transplant 2005: 24: 1431.

22. Szende, A, Williams, A, EuroQol-Group. Measuring Self-Reported Population Health: An International Perspective Based on EQ-5D. Budapest, Hungary: SpringMed Publishing, 2004.

23. Herdman, M, Gudex, C, Lloyd, A et al. Development and preliminary testing of the five-level version of EQ-5D (EQ-5D-5L). Qual Life Res 2011: 20: 1727.

24. Konig, HH, Bernert, S, Angermeyer, MC et al. Comparison of population health status in six European countries: results of a representative survey using the EQ-5D questionnaire. Med Care 2009: 47: 255

25. Petroski, RA, Grady, KL, Rodgers, $\mathrm{S}$ et al. Quality of life in adult survivors greater than 10 years after pediatric heart transplantation. J Heart Lung Transplant 2009: $28: 661$.

26. Grady, KL, Wang, E, Higgins, R et al. Symptom frequency and distress from 5 to 10 years after heart transplantation. J Heart Lung Transplant 2009: 28: 759.

27. Dew, MA, Myaskovsky, L, Dimartini, AF, Switzer, GE, Schulberg, HC, Kormos, RL. Onset, timing and risk for depression and anxiety in family caregivers to heart transplant recipients. Psychol Med 2004: 34: 1065.

28. Sadala, ML, Stolf, NG, Bocchi, EA, Bicudo, MA. Caring for heart transplant recipients: the lived experience of primary caregivers. Heart Lung 2012: 12: 10. 